\title{
Crowdsourcing as a Way to Improve Coastline Conditions Sharing
}

\author{
Carlos Serrão ${ }^{1}$, Helder Carvalho ${ }^{2}$, Nelson Carvalho ${ }^{3}$ \\ Department of Information Science and Technology, School of Technology and \\ Architecture \\ ISCTE-IUL/ISTAR-IUL \\ Av. das Forças Armadas, 1649-026, Lisbon, Portugal
}

\begin{abstract}
Currently, a large number of coastline activities (sports and leisure) exist and host a large number of supporters (growing daily). The number of commercial organizations, such as sport initiation and improving schools that explore such type of activities are also increasing. These kinds of costline activities are dependent of a set of perfect conditions (mostly sun, wind and waves) that affect the type of activity - mostly sportive - that can be conducted (surf, windsurf, paddle, kite surfing, canoeing, paragliding and others) on a given location at a specific time. This article presents and describes a software-based platform - SiUinde - that was developed to capture the power of a large community of interest in the reporting and sharing of coastline conditions, using a smartphone or tablet, and allowing other community users to have an in-depth and timely overview of the best places to enjoy their activities. This crowdsourcing platform uses collaborative intelligence to provide end-users the best possible recommendations for their preferred coastline activities.
\end{abstract}

\section{Introduction}

A large part of our planet is composed by water. About $71 \%$ of the earth's surface is covered by water and the oceans hold around $97 \%$ of earth's water. This makes our planet filled with large coastlines. In Portugal, the coast extends for around $943 \mathrm{Km}$ in the continent adding to $667 \mathrm{Km}$ and $250 \mathrm{Km}$ in Azores and Madeira islands, respectively [1]. Also, other countries, such as Brazil, have more than $8000 \mathrm{Km}$ of coastline, one of the largest in the World. These are just two small samples of what is the importance and dimension of that coastline. Along this massive coastline, communities take advantage from those resources to carry their economical, sportive and leisure activities, all around the year [17].

Currently, there are a large number of near-theshore activities (both in terms of sports and leisure) that engage a large set of practitioners (and the number increases on a daily basis). Indirectly, the volume of commercial companies, such as sport initiation schools, bars and restaurants that explore such type of activities are also rising. These coastline activities are dependent of a set of conditions (in particular on what concerns the sun, wind and swell) that affect the type of activity - mostly sportive that can be carried (surf, windsurf, paddle, kite surfing, canoeing, paragliding and others) on a given location at a specific time.

Considering that the users often spend a large amount of their time finding the perfect spot, that gathers all the right conditions to conduct their favourite near-the-shore activities (or even those users that use the coast and sea as a mean of personal sustenance), emerged the idea to create a system that allows community-oriented sharing of coastline conditions [2] (mostly sun, wind and sea conditions) in a fast, intuitive and interactive manner. Sun, wind and sea conditions are some of the most important parametric conditions to consider, and current systems associated with these types of near-the-shore activities, namely sports, have serious limitations on what concerns the collaborative sharing of reporting information about the specific conditions that are experienced by other users at a specific place and time - most of the existing solutions do not advantage from an immense community of interest that can improve report information mechanisms.

Existing systems are mostly based on meteorologic data or on video feeds that are streamed over the Internet from a particular location. However, the information richness can be higher if users could rely on each other opinions to make better decisions. For instance, in most current applications, users cannot request or view other user's opinions in order to make better informed decisions on the best possible conditions for a specific coastline activity at a given instant, or even, share ideas related to a favourite activity.

Social networking technologies have been revolutionising the way people communicate and build new relations. The functionalities that are currently offered by the usage of social networks can be explored to extract specific knowledge and to add value for end-users. This way, information that is exchanged by the different users on the network can be dynamic and frequently updated. The main idea behind the development of this system, is to have a mechanisms that explores crowdsourcing mechanics 
[19] to create and share conditions reports in order to complement the predictions that are made by traditional existing systems, because on a sufficiently enriched network, there can be constant information exchange, which means that the whole system is always updated. On the other hand, it is possible to use machine learning techniques and data analysis to make increasingly accurate system reports and to produce better recommendations.

The developed system, named as SiUinde (an acronym which resulted from combination of "sea" and "wind" words) can be described as a system that offers a large user community a way to exchange reports and information about the status conditions of the sun, sea and wind (as well as a set of other parameters along the coastline). The user report sharing and access is conducted through mobile devices with available Internet connection. to produce reports on the currently observed conditions at a given location in a given time. Additionally, a user wishing to find a good spot, with the ideal conditions to practice his favourite near-the-shore activity, can, through SiUinde, conduct a survey and analyse reports that were submitted by other users (there is also the possibility of asking the local conditions for certain groups of users, maintain the ranking of the various users, and many others).

In this paper we will provide a description of the developed system, presenting the main components of the solution and how they interact with each other to provide the require functionalities and address major requirements. In following section we provide an overview of the system, its main functionalities and implementation details. Next, another section will present the validation of the developed system prototype. Finally, some conclusions of this work are highlighted and also some directions for future work are presented.

\section{SiUinde, the collaborative platform for sharing of near-the-shore conditions}

There is a immense user community that share a common passion for coastline activities. This community strives to find the perfect place at the right time to conduct their activities, mostly sportrelated.

The main goal of the developed platform was, but not limited to, to provide an immediate solution to some of the solicitations of the near-the-shore activity practitioners, mainly sport active users. Their main request was to have a simple way to access accurate reports about the conditions for a given activity on a specific spot at a given time [3]. Also, they would like to have a platform that could allow them to get recommendations about the best places to practice their coastline activities, and finally they would like the system to be extremely simple to use [20].
Since social networks have become an essential part of everyone everyday life's [4] it has become natural to access them on a computer or on a mobile device. Social networks have made possible for users to promote their public profiles, articulate a list of other connected users, and share content over a intensively networked ecosystem. The developed system takes full advantage of the social network functionalities to:

- Facilitate conditions status reports exchange between the participating users;

- Allow faster information updates for the users;

- Submitted reports can be easily indexed and searched, making them more visible for the endusers;

- Create relationships between different users and allow the possibility of following specific users as preferred information sources on a specific event or activity;

- Facilitate the discovery of other participating users with similar interests or at a specific defined location;

- Create mechanisms for making easier the information exchange between users that share the same interests about a particular activity (for instance, for surf, users can exchange information about materials they use for the different conditions they find on different locations).

As it is possible to conclude, social networks are a the core of the SiUinde system. They represent an important part of the system offering a powerful way to leverage its usefulness and relevance for end-users that share the same interests.

\subsection{Analysis of existing similar systems and platforms}

There are already some existing systems and platforms that are similar to the one being proposed and developed. For this work, we have identified a large set of systems that fall in the same category of the SiUinde system however, most of them had some kind of limitation that we would like to overcome with the new system development. Throughout the analysis that was conducted to this existing systems, it was possible to identify three distinct groups of systems: web-based, native mobile and hybrid mobile applications. It was possible to conclude that all of the selected systems that were considered for analysis, shared a common feature - the access to several coastal condition parameters or other relevant information.

In the web-based system category, Meteopraias (http://www.meteopraias.com/), $\quad$ SurfTotal (http://www.surftotal.com/), BodyBoardTotal, PraiaSAPO (http://praias.sapo.pt/) and BeachCam (http://beachcam.sapo.pt/) were considered in our analysis. The systems in this category depend on a 
web-browser to work and are mostly web sites containing information about the conditions that can be found on a specific location. In the native mobile applications segment, Weddar (http://www.weddar.com/), MSW Surf Forecast (http://magicseaweed.com/) and Glassy Pro Time To Surf (https://glassy.pro/) among the many existing applications, were selected for analysis. Native mobile segment considered the applications that could be installed from one of the existing mobile applications stores. Finally in the hybrid category, Windguru (http://www.windguru.cz/int/), BeachCam, Surf Report (http://www.surfreport.pt/), Vodafone Praia Direto (http://praiaemdirecto.com/), WindFinder (http://www.windfinder.com/) and Surfline (http://www.surfline.com/) were considered for analysis. This category referred to the systems that were a mix between a pure web application and a mobile application - they could be run inside a traditional web browser or installed through a mobile application store.

In order to conduct an analysis of the selected systems and reach some conclusions the following criteria was used:

- Wind information: information about wind speed and wind direction;

- Sea information: factors like the wave size, swell direction, wave periodicity and tide prediction;

- Spot information: information about the number of persons on the water;

- Livecam feed information: information about the number of spots covered and availability periods;

- Information updates: the frequency of information updates on the system;

- Social integration: information abou the users interaction and network creation.

From this analysis it was possible to conclude that most of the existing tools/systems were extremely vertical - most of them offer support for just a type of activity while some give more importance to some specific characteristics over others. It is also possible to conclude that most tools put a major emphasis on the sea and wind conditions (in a static fashion), disregarding other aspects like beach information, livecam feed information and social integration and interaction. None of the systems considered for analysis was using crowdsourcing as a way to collect real time reporting information from users for a multiplicity of coastline activities.

\subsection{Characteristics and requirements}

In the previous section of this article it was possible to identify a set of systems with some commonalities with the SiUinde system. After the identifications and analysis conducted it was possible to identify systems with similar objectives. A set of key-characteristics were identified, as presented in the previous section, and a comparison was performed between the different tools according to those characteristics, in terms of advantages and disadvantages. Due to the defined objectives for SiUinde, the list of key-requirements that platform should have to support, include reporting simplicity and easiness of usage, integration with major social networks, production of personalised recommendations and self-learning capabilities.

The list of requirements for a system like SiUinde is vast and long however, it is possible to highlight some of the more relevant ones:

- The system has to provide the user access to reports and and creation of reports about the conditions of a specific near-the-shore georeferenced spot (containing a set of parameters sun, wind, wave, affluence, and other conditions;

- It is also important to have the possibility to write and receive comments on reports, and to add complementary information to such reports (such as photos and short videos, that are directly related with the report);

- The the design and implementation of a simple, intuitive and easy to use reporting scale, to report conditions on particular coastline location (auto-determined by the user location), and provide advices based on the type of activities that might be more advisable to practice on such location;

- Allow the users to ask for specific geo-located conditions, by sending a request to specific users or to the "network" - these requests will be sent to the users that are on that location or nearby;

- Create system "gamification" functionalities [5][6][7] to allow the establishment of a ranking that awards the best submitted user reports and recommendations and, the creation of virtual geocaching [8] functionalities that would reward users with points for being at a specific location or by sharing the discovery of new spots;

- Multi-platform, offering support for both iOS and Android (two of the most common mobile platforms, that dominate the mobile applications market);

- Design a mechanism that allows the crossing of a set of different sources of information (using some existing publicly available API) to be capable of producing reliable recommendations for users on what concerns the optimal conditions to endure its favourite near-the-shore activities, in a specific geographical range;

- Analyse the system information and find relations between the data that allows the prediction of ideal conditions for a given type of activity in a given location;

- Develop a way so that the system could learn from the user system usage (types of activities, activity on the system, interaction with other users, other) and user preferences [9] to automatically create personalised near-the-shore 
routes that are in line with the user requirements [10]. For instance, it will be possible for the system to create a specific route for surf spots, in a specific region, that are adequate for a specific user skill-level.

Having into consideration of the different requirements that were identified previously, the SiUinde platform system was developed. The prototype took into consideration not only the enlisted requirements but also some of the characteristics of the applications that were previously identified and presented.

\subsection{Mobile application}

The SiUinde platform is composed by two major components that allow the implementation of the desired functionalities described on the listed requirements: a mobile front-end and a server-side backend (following a similar paradigm to what is the current state of the art in terms of mobile development).

During the different iterations of the development of the SiUinde mobile solution, it was possible to conceptualise the final results through the conceptions of several screen mockups and storyboards that allowed the realisation of how the final system would look like and the different interactions between the multiples screens (Figure 1). These mockups and storyboards were used to guideline the implementation of the final prototype.

The development of the mobile application also placed an important emphasis on the UI/UX [16] aspects - the objective overall objective was to build an captivating user experience for the end user that would be extremely easy to use in order to facilitate both the reporting communication and sharing as well as the visualisation of geo-referenced reports (Figure 2).

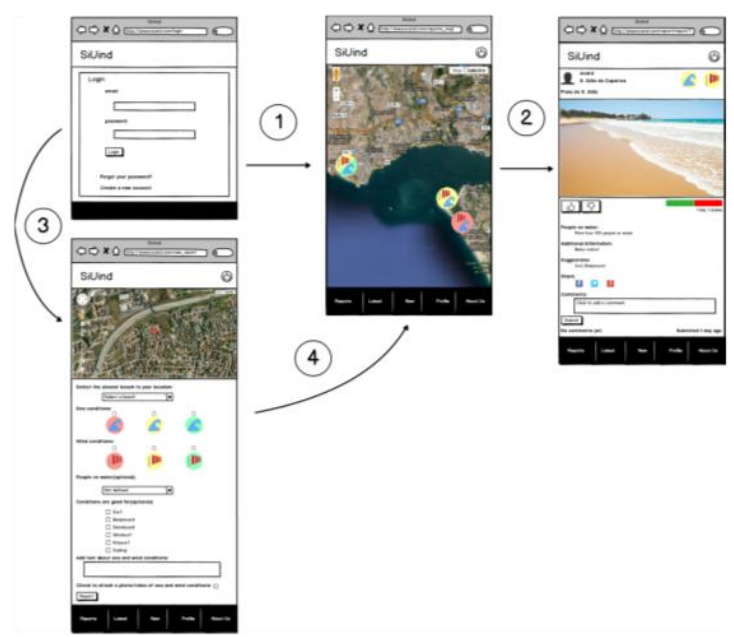

Figure 1. Some of the mockups and storyboards of the developed SiUinde mobile application prototype.

These mockups and storyboards were later on implemented on the system prototype
In order to facilitate the usage of this mobile application, a simplistic graphical iconic scale was created to represent most of the reporting parameters that SiUinde would have to handle (sun, wind, wave, others). The application detects the user location when producing a report, and the user with some simple touches on the screen can create in a fast manner a simplistic report and share it with the community - it is also possible to add further details to its report, when submitting it or at a latter stage.

One of the major concerns in the design of the mobile front-end was to ensure that the mobile application was as much as possible platform independent (Figure 1). On a first stage, the first version of the SiUinde prototype was developed using Web-based technology (HTML5, CSS e Javascript) [11] which allowed the application to run inside any mobile web browser.

After this first version of the prototype was concluded some other options were considered - like using Phonegap [12] or natively write the mobile application on iOS (using ObjectiveC) [13] and Android (using Java) [14]. Finally, a third option was followed, allowing SiUinde application to obtain mobile platform independence, while producing native applications for both iOS and Android. For the development of the mobile front-end, it was selected a hybrid mobile development approach based on a framework called Appcelerator Titanium [15][18]. This Appcelerator Titanium framework follows an Model-View-Controller (MVC) development approach, where the controllers and models are developed using Javascript, while the views are easily created with XML and TSS (which represents a role quite similar to the one CSS represents in HTML).

After the source code development using this approach and languages, Titanium then generates native applications for different mobile platforms (in this case, iOS and Android) - Titanium does not yet supports Windows Phone, but is working on developing support for that platform in a near future.

In the mobile application it will be possible to add some extra information to the report, such as free description text, comments, pictures or even short videos. The same iconic scale is used to place icons over the map, representing the different conditions experienced at different locations. 


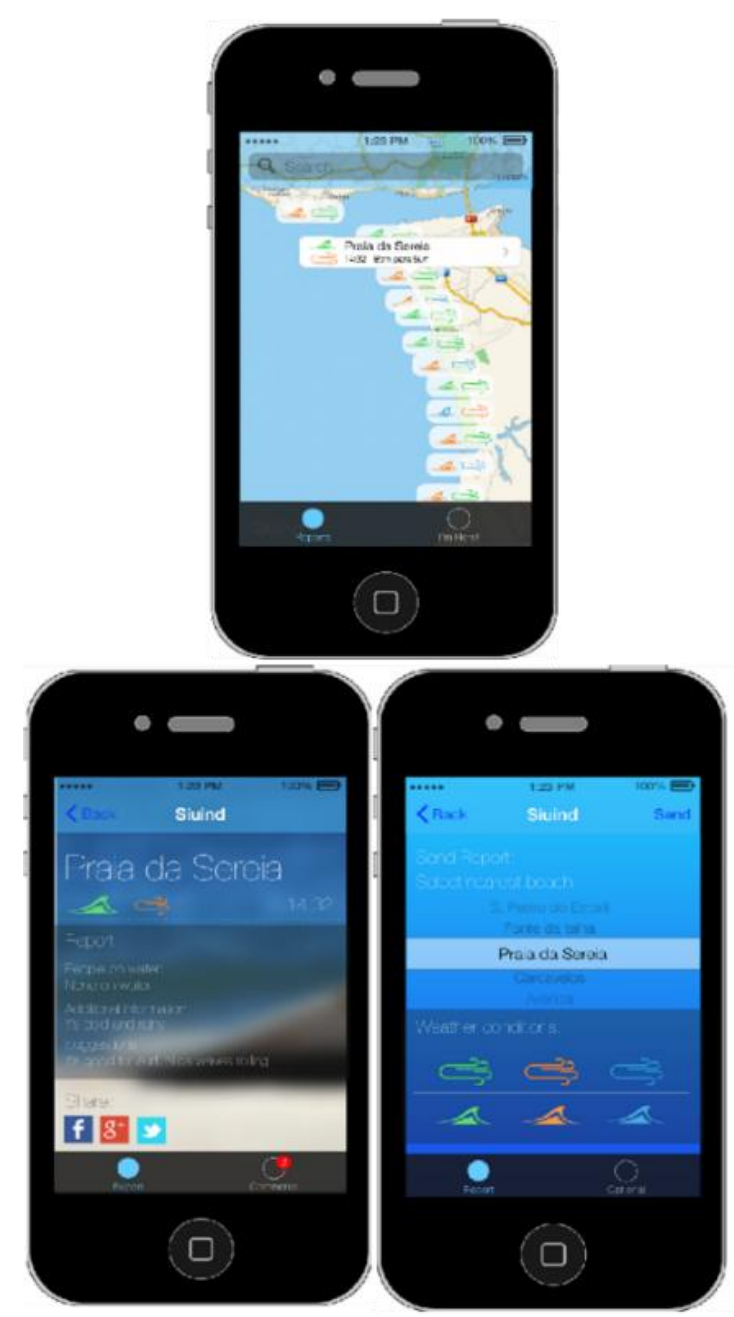

Figure 2. Som of the SiUinde mobile application screenshots running on iOS (in one of the screenshots is possible to visualize the iconic scale that was developed and used on the reports and on the map)

From this, it is important to notice that a lot of effort has been placed on the development of a mobile application that was at the same time attractive and easy to use, but also quite useful for the target users and community. The reporting and sharing mechanisms, allied with the reporting simplicity, are the top of the key-functionalities that make the difference to other existing applications.

\subsection{Backend system}

As with any other mobile-based application, part of the logic behind the application resides on the server-side backend. The SiUinde system is no exception. The SiUinde backend platform is responsible for the implementation of many of the tasks that the mobile application will have to make available for the end-user.

The SiUinde mobile application front-end that was presented in the previous sections, interacts with the backend through a well-defined REST API. This API provides the only secure and authenticated communication channel between the mobile application and the backend.

This API offers access to a different set of functionalities that cover the report collection and generation, social network report sharing and commenting, user registration and authentication (acting as a broker to social networks), recommendations, access to complementary information and reports classification and feedback.

All of this modules are presented in the following image (Figure 3). This image provides an overview of the backend architecture, initially developed in PHP and later ported to Node.js, due to scalability and future development purposes. When compared to PHP, Node.js is clearly gaining momentum. Node.js is based on JavaScript, providing an improved, functional and non-blocking I/O mechanism that allows better performance, and allowing the usage of the same development language, Javascript, on both end of the solution.

On top of the SiUinde backend architecture, a REST API implements a secure and authenticated channel between the mobile application and the backend. This REST API implements the functions that are used invoke internal functionalities on the backend. These functionalities allow to the SiUinde mobile application to implement a significative part of the previously identified requirements.

One of the blocks of the backend is the user information module. This user information module is responsible for handling with the registration of the users on the system (including the possibility of the association of the user social network accounts, and use the integrated social network authentication), handle the authentication requests from the mobile application, the storage and retrieval of information about the user and their relations relations with other users on the system. To sum up, this module is responsible for the handling of all the user-related information and user interaction on the system (with other users or with other elements of the system). This module is also deeply integrated with the reporting module. This allows the system to follow the user-generated reports, collect their feedback and establish a user ranking system based on their peerrecognition on the system - based on the opinions of other users.

Another relevant module on the system is the social network interface module. The role of the social network interface module is to ensure that the social network liaison mechanisms are in place in order to retrieve user-related information of the social networks on behalf of a given user and also to ensure that it is possible to publish and share information about the user-generated reports and associated content on a set of previously user preselected platforms. This module assures the entire responsibility of handling the necessary communication with the selected social network platforms and increase community network effects. 


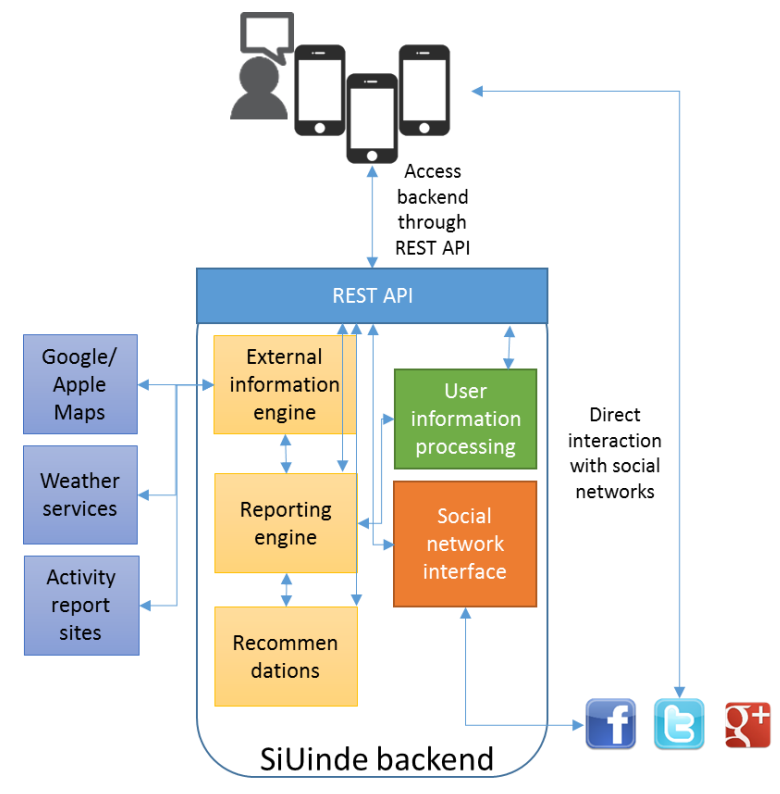

Figure 3. General overview of the SiUinde backend architecture and associated services

The acquisition of different data from external information sources is the responsibility of the external information engine module. This external information engine module, is responsible for establishing the connection with SiUinde's external sources of data, such as Google maps and Apple maps (it depends on the mobile platform, Google Maps for Android and Apple Maps for iOS), open weather services (we are currently using OpenWeatherMaps, however other weather services can be integrated in future releases of the system) and other near-the-shore activity sites (which provide open access to data and open APIs) to feed activity specific information into the SiUinde system. For instance, it will be possible to integrate news about specific sportive modalities on the system.

Another important module on the backend system is the reporting engine module. This reporting engine module aggregates the reporting information collected from the users on the community, combines the different user reports and the feedback, and presents the different reports on the maps. The reporting engine module also combines the reports with external information sources, with information from the users and from the recommendations engine module. This module is used to contextualize the reports of the maps that are presented to the endusers.

Finally, another of the modules on the backend is the recommendations engine module. This module is responsible for gathering all the information on the system for a given user, analysing its preferences and activities (and the activities of its friends), their reports, comments and feedback to produce specialised and personalised recommendations for the user. This recommendations module is one of the key differentiation aspects on the system, when compared with others.
The backend is of extreme importance for the SiUinde system, in particular for the mobile application. A significant part of functionalities that are present at the mobile front-end are the result of the work being processed by this backend. The different modules that compose the backend system are responsible for the implementation of some of the most important requirements of the SiUinde system.

After the development of the SiUinde prototype it was important to assess the system and evaluate the user's opinions about the system. In the section that follows, some testing and validation results of the system are presented, which constitute an important source of feedback to document if the SiUinde requirements match the users expectations and also to identify opportunities for improvement and future work.

\section{System testing and validation}

Testing and validating the system developed was another important step to verify its quality and acceptance. After the SiUinde prototype has been developed and some internal tests conducted, a group of users were selected in order to conduct real-world testings with it (the survey was conducted in Portugal, due to the intrinsic characteristics of the system and the conditions of development). Due to the requirements of the developed system, two different groups were selected for testing: a first group, composed by users that were our major target (users that carry some type of coastline activities) and a second group containing non-expert type of users.

The objective of this testing phase was to validate if the developed prototype was indeed addressing the requirements that were identified as major concerns of these group of users and how they evaluated the system in general and the mobile application in particular. The users were given the opportunity to freely test the SiUinde mobile application and in the end they were invited to fill a small survey about their experience.

The survey that was conducted for validation of the SiUinde prototype system targeted a group of users that focused primarily in one of the most important features of the system: the reporting and sharing mechanisms. That survey was composed by five different groups of questions: a description and identification of the users, the type of coastline activities and the existing applications, the reports communication and sharing on the system and social networks propagation, the reports visualisation and a general evaluation of the system from the perceived value of the users. 


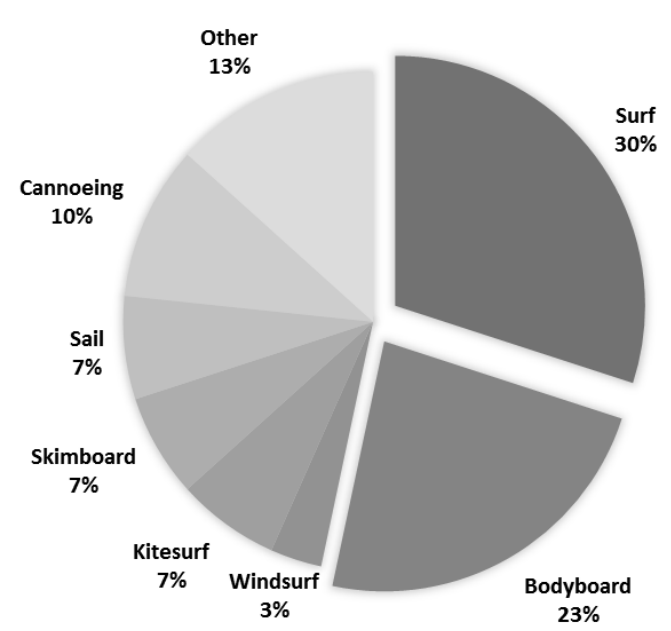

Figure 4. The following graphic represents the type of sports that are practiced by the users that participated in the

\subsection{Users presentation and description}

There were a total of 250 participants in the tests and validation conducted to this prototype stage of the system. From these 250 participants, $75 \%$ were male and $25 \%$ female, around $80 \%$ of the respondents had between 21 and 40 years old and $65 \%$ of them practice some type of near-the-shore sports activity (Figure 4). From that sample it is also important to conclude that most of the users were focused on surf (30\%) and bodyboard (23\%) activities. Nevertheless there are almost $47 \%$ of users that conduct some other type of near-the-shore activities, proofing that there is also a large number of potential interest in SiUinde beside the surfers and bodyboarders communities (for which there are already a large number of applications available).

The remaining $35 \%$ of the respondents that didn't practice any near-the-shore sportive activity, correspond to the users that are still interested in knowing coastline conditions, for other purposes: pleasure, fishing, economical activities or other.

\subsection{Coastline activities and applications}

The next important stage of the conducted validation was to assess if the users were aware of some other similar existing applications, and if they were, it would be extremely valuable to know exactly what was the users opinions about those applications.

Therefore, it was possible to conclude that around $61 \%$ of the surveyed users used some kind of application to find information about the near-theshore conditions and that Windguru web-based service was selected as their main application of choice in $46 \%$ of the cases. The user satisfaction level with the type and quality of the existing applications is high (83\% of the users were quite satisfied), however none of the user gave top scores to the existing applications, referring the need for improvement. Also, on what concerns the information actuality on the analysed applications, around $40 \%$ of the respondents rated this aspect with a medium grade (3, on a scale from 1 to 5 ).

From this, it is important to conclude that users find extremely relevante using some kind of mobile applications to find about the conditions on the coastline. It is also important to notice that although users are usually satisfied with their current application, they refer that there is room for improving existing applications.

\subsection{Reports sharing}

The following part of the validation refers to one of the most critical characteristics of these type of systems, and consequently also of SiUinde - reports generation and sharing.

The results presented here represent the SiUinde prototype application usage by the surveyed users. During the tests and application testing with users it was possible to extract some conclusion from the analysis conducted. These results can be resumed in the following:

- A large majority of users $(86 \%)$ agree that it is really easy to find their position on the map that is presented by the mobile application;

- $77 \%$ of the users attested that the geolocation on the application was accurate and precise, allowing the application to accurately detect the position of the user on the map;

- The functionality that allows the users to select the nearest beach was considered as extremely useful and intuitive. $75 \%$ of the respondents of the respondents considered that this was a useful;

- $81 \%$ of the users considered that the amount of beaches presented on the map was sufficient to make the application useful;

- Around $72 \%$ of the respondents considered that the icons created and the scale selected to produce and visualize the reports (in terms of sun, sea and wind conditions) were quite adequate and intuitive;

- On what concerns the reporting on the number of persons on the water, $61 \%$ of the respondents consider this functionality extremely useful and intuitive to use;

- Regarding the selection of activities according to the conditions of the selected location, $79 \%$ of the respondent users considered that this functionality was useful and intuitive;

- The functionality to add free text, photos and video to the report, has been well accepted by end-users and $96 \%$ of the users highly valuated this option;

- The easiness about report submitting and visualisation of reporting information on the 
maps has also received good feedback $-98 \%$ of the users were satisfied with these functionalities.

From this section it possible to easily conclude that the users that evaluated our system and responded to the questionary, highly value the solutions that were adopted and implemented for the reports visualisation and reports submission, as two of the major functionalities of the SiUinde prototype system.

\subsection{Overall evaluation of the system}

After all the development that was accomplished on the development of the prototype, it was validated and some tests concluded and results collected.

On what concerns the general usage of the SiUinde application by end-users, $76 \%$ of the users considered that the application was extremely easy to use. Also, $76 \%$ of the users considered that SiUinde introduces something new when compared with the similar applications that they already use and that are available on app stores and on the web.

Another important aspect from this part of the survey is the fact that $85 \%$ of the respondent users considered using SiUinde in the near future, replacing their current applications of choice.

In resume, as a general conclusion from this validation and testing, it was possible to conclude that users find the application useful to support their near-the-shore activities, easy to use and attractive, capable of providing intuitive reporting and visualisation functionalities, and would consider using it in the future.

Although still on a prototype stage, the user reaction to the usage of the application was quite stimulating, revealing the interest of the users on its usage.

\section{Conclusions}

During the present article it was presented a system that was developed to allow the users to collaborate in the sharing of information about a group of parameters that are important for a set of coastline activities.

Coastline activities, mostly sport activities, are gaining importance among users, and more communities of users engage in some type of activities and search and exchange information about those activities.

The developed solution, SiUinde represents an end-to-end solution, supported on a mobile smartphone/tablet application and an integrated backend system, which collects reports from individual users and from other external services, and allows users, according to their specific preferences and activities, to be informed about the near-the-shore conditions in a given place and at a given time.
In addition, this article has also reported on the work that was performed to validate SiUinde with end-users. In order to achieve this, it was conducted a specific survey with a group of potentially interested users. Due to initial the nature of the work conducted and its characteristics only Portuguese test-users were considered.

From the survey that was conducted, and its subsequent analysis, it was possible to conclude that,in general, users were satisfied with the functionalities present on the application prototype and are willing to use the SiUinde application in the near future, as their top application of choice.

Although promising, there is still a larger validation that needs to be conducted after the public release of the system. Moreover, some functionalities, like the recommendation still need to be improved to meet the user demand.

Once again it is clear that this type of systems can be quite useful for end-users, in particular those that spend some precious time looking for the perfect spot and time to conduct their preferred near-theshore activities. SiUinde presents itself as an interesting alternative to all of them.

\section{References}

[1] S. P. Srivastava, H. Schouten, W. R. Roest, K. D. Klitgord, L. C. Kovacs, J. Verhoef, and R. Macnab, "Iberian plate kinematics: a jumping plate boundary between Eurasia and Africa," Nature, vol. 344, no. 6268, pp. 756-759, Apr. 1990.

[2] B. Libert and J. Spector, We Are Smarter Than Me: How to Unleash the Power of Crowds in Your Business. Wharton School Publishing, 2007, p. 176.

[3] Network Strategies in Europe: Developing the Future for Transport and ICT. Ashgate Publishing, Ltd., 2008, p. 303.

[4] Danah M. Boyd and N. B. Ellison, "Social Network Sites: Definition, History, and Scholarship," J. Comput. Commun., vol. 13, no. 1, pp. 210-230, Oct. 2007.

[5] G. Zichermann and C. Cunningham, Gamification by Design: Implementing Game Mechanics in Web and Mobile Apps. "O’Reilly Media, Inc.," 2011, p. 208.

[6] K. Huotari and J. Hamari, "Defining gamification," in Proceeding of the 16th International Academic MindTrek Conference on - MindTrek '12, 2012, p. 17.

[7] S. Deterding, D. Dixon, R. Khaled, and L. Nacke, "From game design elements to gamefulness," in Proceedings of the 15th International Academic MindTrek Conference on Envisioning Future Media Environments MindTrek '11, 2011, p. 9.

[8] C. J. Hooper and J. W. Rettberg, "Experiences with Geographical Collaborative Systems: Playfulness in Geosocial Networks and Geocaching." 01-Aug-2011.

[9] T. Segaran, Programming Collective Intelligence: Building Smart Web 2.0 Applications. "O'Reilly Media, Inc.," 2007, p. 360.

[10] Information Visualization in Data Mining and Knowledge Discovery. Morgan Kaufmann, 2002, p. 407.

[11] R. F. Grove, Web-Based Application Development. Jones \& Bartlett Publishers, 2009.

[12] J. M. Wargo, PhoneGap Essentials: Building CrossPlatform Mobile Apps. Addison-Wesley, 2012, p. 384. 
[13] S. G. Kochan, Programming in Objective-C: Updated for iOS 5 and Automatic Reference Counting (ARC) (Developer's Library). Addison Wesley, 2011, p. 560.

[14] J. Steele and N. To, The Android Developer's Cookbook: Building Applications with the Android SDK (Developer's Library). Addison Wesley, 2010, p. 400.

[15] C. Brousseau, Creating Mobile Apps with Appcelerator Titanium [Paperback]. Packt Publishing, 2013, p. 318.

[16] M. David and C. Murman, Designing Apps for Success: Developing Consistent App Design Practices. CRC Press, 2014, p. 280.

[17] Bolle, A., Mercelis, P., Roelvink, D., Haerens, P., \& Trouw, K. (2011). Application and validation of XBeach for three different field sites. Coastal Engineering Proceedings, 1(32), sediment- 40 .

[18] Pollentine, B. (2011). Appcelerator Titanium Smartphone App Development Cookbook. Packt Publishing Ltd.

[19] Perin, C., Vuillemot, R., \& Fekete, J. D. (2013, October). Real-Time Crowdsourcing of Detailed Soccer Data. In What's the score? The 1st Workshop on Sports Data Visualization.

[20] Woerndl, W., Schueller, C., \& Wojtech, R. (2007, April). A hybrid recommender system for context-aware recommendations of mobile applications. In Data Engineering Workshop, 2007 IEEE 23rd International Conference on (pp. 871-878). IEEE. 